\title{
THE REGULATION OF EYE GROWTH AND REFRACTIVE STATE: AN EXPERIMENTAL STUDY OF EMMETROPIZATION
}

\author{
DAVID Trollo* and Josh WALlMaN \\ Department of Biology, City College of the New York, New York, NY 10031, U.S.A.
}

(Received 12 March 1990; in revised form 19 November 1990)

\begin{abstract}
During growth the vertebrate eye achieves a close match between the power of its optics and its axial length with the result that images are focused on the retina without accommodative effort (emmetropia). The possibility that vision is required for the regulation of eye growth was studied experimentally in chicks made myopic or hyperopic by different visual manipulations. After discontinuing these visual manipulations, the eyes returned quickly to emmetropia mainly by adjusting the growth of their vitreous chambers; growth stopped in eyes recovering from myopia and continued in eyes recovering from hyperopia. Because both hyperopic and myopic eyes were already larger than normal controls, the difference in growth indicates that refractive error, rather than eye size per se, guides the eye toward emmetropia. Evidence is also presented for nonvisual shape-related control of eye growth, but this is slow-acting and cannot explain the emmetropization from induced refractive errors. Both the visually guided and shape-related mechanisms work even in eyes with the optic nerve cut, indicating that the two mechanisms are local to the eye. Although the optic-nerve-sectioned eye can sense the sign of a refractive error and initially adjust growth accordingly, it eventually overshoots emmetropia and reverses the sign of the initial refractive error. Whether this is due to loss of feedback from the central nervous system or retinal ganglion cells is unclear.
\end{abstract}

Chicks Emmetropization Eye growth Hyperopia Myopia Refractive state

\section{INTRODUCTION}

The eyes of neonates of a variety of species have refractive errors (generally hyperopia) that tend to diminish with time (e.g. McBrien \& Norton, 1987; Mohindra \& Held, 1981; Wallman, Adams \& Trachtman, 1981a). This growth from ametropia toward emmetropia is commonly referred to as emmetropization, although there is little agreement on what developmental processes are behind it (for summaries see: Borish, 1970; McBrien \& Barnes, 1984; Morgan, 1967). One controversial view is that eye growth is actively regulated, and that visual experience guides refractive state toward emmetropia (e.g. Banks, 1980; Medina, 1987a, b; van Alphen, 1961; Young, 1977). Alternatively, nonvisual explanations exist; it has been argued that emmetropization is the postnatal continuation of genetically determined embryological processes that, in combination with the physical constraints on the growing eye, result in normal eye size and shape (e.g. Mark, 1972; Sorsby, 1979).

*Present address: Section of Neurobiology and Behavior, Cornell University, Ithaca, NY 14853, U.S.A.
Three lines of evidence suggest that emmetropization may be an actively regulated visual process: (1) the development of refractive state in human neonates is very sensitive to visual disturbance (Hoyt, Stone, Fromer \& Billdon, 1981; Nathan, Kiely, Crewther \& Crewther, 1985; O'Leary \& Millodot, 1979; Robb, 1977), and various experimental species deprived of form-vision are known to develop myopia (e.g. Marsh-Tootle \& Norton, 1989; Troilo, Judge, Ridley \& Baker, 1990; Wallman, Turkel \& Trachtman, 1978b; Wiesel \& Raviola, 1977); (2) sustained near vision has long been associated with myopia. In humans, occupations associated with extensive reading or other close work show an increased incidence of myopia (e.g. Donders, 1952; Duke-Elder, 1930; Dunphy, Stoll \& King, 1968; Goldschmidt, 1968; Richler \& Bear, 1980; Young, 1977). In animals, caging or close visual surrounds lead to myopia (Rose, Yinon \& Belkin, 1974; Young, 1961), even when the near environment is restricted to part of the visual field (Miles \& Wallman, 1990); (3) the eyes of chicks made functionally myopic with positive spectacle lenses or hyperopic with negative 
spectacle lenses (Schaeffel, Glasser \& Howland, 1988) change their axial lengths and refractions so that they partially compensate for the errors produced by the lenses; functionally hyperopic eyes grow longer and become myopic, and functionally myopic eyes grow less and become hyperopic.

The question of the mechanism of emmetropization is of more than academic interest; much speculation has been directed at understanding how emmetropization is upset in children resulting in refractive errors, particularly myopia. For example, determining whether eyes attain emmetropia by active visual feedback can provide a rationale for whether or not to intervene in cases of mild refractive errors in children. Controlled experimental investigations have, however, been noticeably absent. The present study shows that eye growth in chicks is under visual feedback control. We studied the growth-regulation of chick eyes by monitoring their growth toward emmetropia from either induced myopia or hyperopia. Because the recovery from induced refractive errors is most rapid and complete in young chicks when growth toward emmetropia is normally observed (Wallman et al., 1981a) we propose that this "experimental emmetropization" may use the same mechanism responsible for normal emmetropization.

Recovery from experimentally induced myopia in chicks has been reported elsewhere (Wallman \& Adams, 1987). When the myopiaproducing visual form deprivation was removed, the eye became less myopic as a result of the cessation of vitreous chamber growth. It is unclear, however, whether the mechanism behind this recovery from myopia is driven by an error signal related to visual cues.

Recovery from induced hyperopia has not been shown prior to this report. Hyperopia can be induced in chicks by raising them in total darkness, and results from a flattening of the cornea in an eye that is actually larger than normal (Gottlieb, Fugate-Wentzek \& Wallman, 1987). In this paper we show that recovery from dark-induced hyperopia occurs as a result of an increase in corneal curvature and vitreous chamber depth. This supports the existence of visual regulation of eye growth because (1) the change in corneal curvature is in the opposite direction of what is normally observed during eye growth, and (2) it involves the further enlargement of an already abnormally enlarged vitreous chamber.
For visual regulation of eye growth to occur there must be a mechanism to sense whether the eye is myopic or hyperopic in order to direct growth appropriately. Because of this, it is not unreasonable to expect that the brain's visual pathways are involved in the feedback loop. The involvement of the brain in the development of myopia resulting from visual deprivation has been questioned by several reports (Raviola \& Wiesel, 1985; Troilo, Gottlieb \& Wallman, 1987; Wildsoet \& Pettigrew, 1988), but the role of the putative intraocular eye growth control mechanism in normal growth is unclear. In the present study we used optic nerve section to determine the level of visual processing necessary for recovery from induced refractive error.

\section{METHODS}

White Leghorn chicks (Gallus gallus domesticus) were hatched in the laboratory and normally raised in temperature-controlled brooders under fluorescent lights ( $14 \mathrm{hr}$ light/ $10 \mathrm{hr}$ dark).

To induce myopia, chicks had translucent plastic occluders (Wallman, LeDoux \& Friedman, 1978a) secured around the eye with collodion (Fisher Scientific). These occluders had a portion cut away that enabled form vision in the frontal monocular visual field (approx. $65 \mathrm{deg}$ ), leaving the lateral visual field occluded (i.e. the temporal retina viewed the frontal visual field while the nasal retina viewed the white matte inside of the occluder). Although this technique produces maximal myopia in the nasal retina (Wallmann, Gottlieb, Rajaram \& Fugate-Wentzek, 1987), the eyes were always enlarged and myopic in the central retina around the optic axis where all our measurements were made. The partial visual deprivation permitted daily monitoring of the eyes after surgery.

To induce hyperopia, chicks were raised from hatching to 4 weeks in complete darkness inside a light-proof, temperature-controlled chamber. During the first week of dark-rearing the chicks were force-fed 3-4 times daily a suspension of pulverized chicken feed in water. (During feedings a dim red light was present for less than a minute.) After a week of force-feeding the chicks learned to eat and drink in the dark, and at the end of 4 weeks their weights were not significantly different from those of normally reared chicks. 
All measurements were performed on chicks lightly anesthetized with a mixture of chloral hydrate and sodium pentobarbital (Chloropent, Fort Dodge; $0.25 \mathrm{~cm}^{3} / 100 \mathrm{~g}$ i.m.), and with cycloplegia produced by a curare solution (Wallmann \& Adams, 1987). The chicks typically recovered fully from the anesthesia and mydriasis within about $5 \mathrm{hr}$. The chicks usually underwent the following set of measurements taken along the optic axis of both eyes: refractive state was determined by refractometry,* corneal curvature by keratometry, and the axial dimension of the eye and its components were measured by A-scan ultrasonography (for details see Wallman \& Adams, 1987).

\section{Recovery from induced refractive errors}

Emmetropization was examined experimentally by assessing the ability of eyes to correct an induced refractive error after the visual manipulation causing it was discontinued. 16 chicks were used in this study. 9 chicks were made unilaterally myopic by monocular visual form deprivation, while 7 chicks were made bilaterally hyperopic by raising them in complete darkness. After 2 weeks of the visual form deprivation, or 4 weeks of dark-rearing, the treatments were discontinued and the chicks were returned to normal rearing conditions for 2-3 weeks. Different durations were chosen because they produced approximately the same degree of refractive error. The measurements of refractive state and ocular morphology were performed the day the visual manipulations were discontinued and then again 1, 2 and, in some cases, 3 weeks later. For the statistical comparisons to normal values we used refractions and morphological data from normal chicks at various ages from our earlier experiments. This was necessary because a contralateral control eye is absent in dark-reared chicks.

\footnotetext{
*The artifact of retinoscopy (Glickstein \& Millodot, 1970), which biases refractions in small eyes toward hyperopia, is not accounted for in the measures of refractive error presented throughout this paper. This is because of the difficulties in accurately determining the changing magnitude of the artifact in individual eyes as they grow. Based on retinal thickness and the axial lengths of chick eyes at various lengths, it is estimated that the artifact accounts for approximately 4,3 , and $2 \mathrm{D}$ of hyperopia in the measured refractive errors of normal 2, 4 and 8 week old chicks, respectively (Wallman \& Adams, 1987).
}

\section{Optic nerve section and emmetropization}

In order to begin to test the level of visual processing necessary to sense and respond to induced refractive error the experimental emmetropization paradigm described above was used on chicks with unilateral intra-orbital optic nerve section (see Troilo et al., 1987). Only chicks lacking pupillary responses in the nervecut eye were used.

Chicks with unilateral optic nerve section performed at hatching were made myopic by binocular visual form deprivation $(n=7)$ for 1 week. At that time the occluders were removed and both the optic-nerve-cut eye and its fellow control eye were monitored over an additional period of up to 7 weeks. A second group $(n=8)$ was reared from hatching in complete darkness to produce hyperopia. At 4 weeks of age they were removed from the dark, measured, and each chick received unilateral optic nerve section. After being returned to normal rearing conditions, their eyes were monitored for 3 weeks more. To test whether recovery from ametropia in the optic-nerve-sectioned eyes depended on the fellow eye having a refractive error of the same sign, additional chicks $(n=4)$ received visual deprivation of only the opticnerve-sectioned eye leaving the unoperated eye with normal vision.

\section{RESULTS}

\section{Recovery from induced refractive errors}

The eyes of chicks are able to make the appropriate growth changes to compensate for either induced myopia or hyperopia and thereby grow toward emmetropia. The compensation is largely the result of modulation in the depth of the vitreous chamber, but changes in the anterior segment (i.e. cornea, anterior chamber and lens) toward normal dimensions also occur.

Recovery from myopia. Figure 1 shows that visual deprivation from hatching to 2 weeks of age produces significant myopia relative to the eyes of normal controls (median axial refractions: -7.87 vs $+3.57 \mathrm{D}$ respectively; Mann-Whitney $U$-test, $P<0.01$ ). One week after the visual deprivation was discontinued, the refractive state had changed significantly $(-7.87$ to $+3.96 \mathrm{D}$; Wilcoxon test for paired data, $P<0.01$ ), returning to control levels in all nine cases.

The anatomical correlates of the refractive changes just described are illustrated in Fig. 2A. 


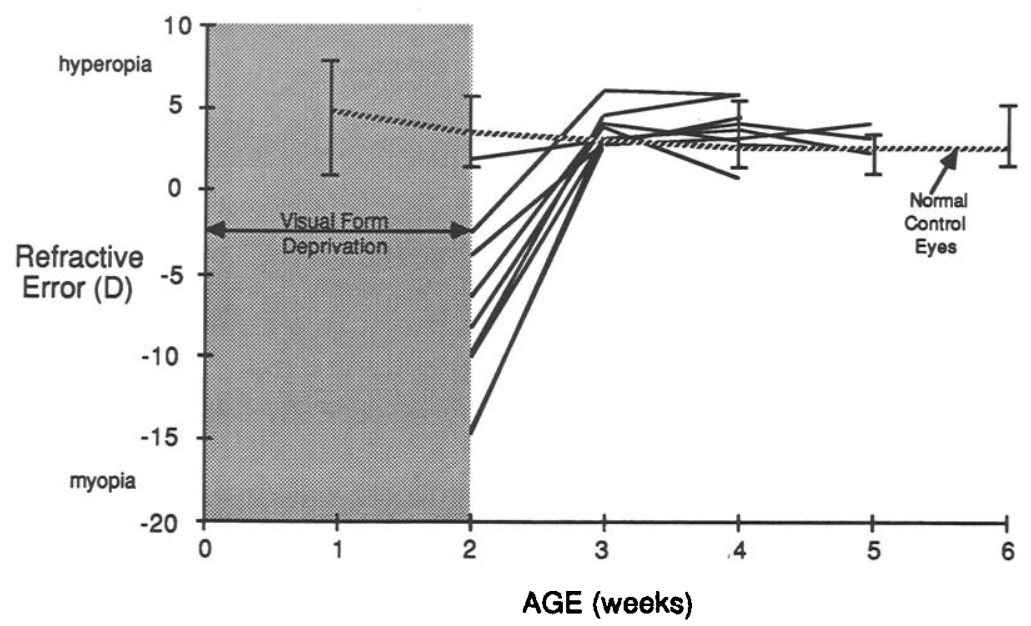

Fig. 1. Chicks made myopic by visual form deprivation recover when the manipulation is discontinued. The individual refractions of chicks reared until 2 weeks of age with visual form deprivation are shown as solid lines. Measurements of refractive error were first made at 2 weeks of age when the occluders were removed, and then at 3,4 and, in some cases, 5 weeks of age. One week after the occluders were removed, the refractions have returned to normal levels. The broken line shows the median refractive errors of normally-reared control chicks; at least 9 chicks were measured at each age shown. Error bars indicate the 10th and 90 th percentiles.

After 2 weeks of visual form deprivation, only the mean vitreous chamber depth differs significantly between deprived and normal eyes (means: 5.66 vs $5.16 \mathrm{~mm}$ respectively; $t$-test, $P<0.01$ ). Following removal of the visual deprivation at 2 weeks the vitreous chamber stopped growing. The mean rate of change in vitreous chamber depth in the myopic eyes between 2 and 3 weeks is significantly less than that in control eyes $(-0.092$ vs $0.281 \mathrm{~mm} /$ week; $t$-test, $P<0.02)$. This resulted in the mean vitreous chamber depth at 3 weeks of age being not significantly different than that measured at 2 weeks ( 5.57 vs $5.66 \mathrm{~mm}$ respectively; paired $t$-test, $P=0.154)$. It is likely that part of the apparent vitreous chamber shortening is the result of concurrent lens growth (the ultrasound measurement of the vitreous chamber is taken from the posterior surface of the lens to the inner surface of the retina). Corneal curvature, lens thickness, and anterior chamber depth were all within normal ranges after 2 weeks of visual deprivation, and continued to grow normally throughout the period when refractive state returned to emmetropia.

To evaluate the influence on refractive state of the changes in the various ocular components between the ages of 2 and 3 weeks (after the visual deprivation had been discontinued and the eyes had returned to emmetropia) we used paraxial ray-tracing to estimate the change in refraction that would result from a given change in an ocular component. Our procedure was carried out for individual eyes as follows. First, because no data for the lens curvatures were available, a lens power was calculated for each eye that reconciled the ocular components with the refractions measured at 3 weeks. Next, one parameter (shown in parentheses in Fig. 2B) was changed either to the value it had had at 2 weeks when the eye was ametropic, or to the mean value of normal eyes at 3 weeks. The resulting refractive error was then calculated for each eye and the median for the 7 eyes plotted in Fig. 2B. If the predicted refractive error was more myopic than the refraction observed, the actual change in the particular component considered had contributed to the recovery from myopia.

As an aid to understanding Fig. 2B, consider for example the second bar from the top (corneal curvature at 2 weeks). If the eyes at 3 weeks had retained the corneal curvature they had at 2 weeks their mean refractive error would have been $-10 \mathrm{D}$, instead of the $+4 \mathrm{D}$ observed. Therefore the actual change in corneal curvature alone made the eyes $14 \mathrm{D}$ less myopic than they would have been without these changes, and so contributed to the recovery from myopia. Figure $2 \mathrm{~B}$ also shows that changes in the anterior chamber depth play a similar, but smaller, role to corneal curvature in the determination of refractive state; if the depth of the anterior chamber had not increased between 2 and 3 weeks, the optical power of the eye would not have been reduced sufficiently to achieve the 
A

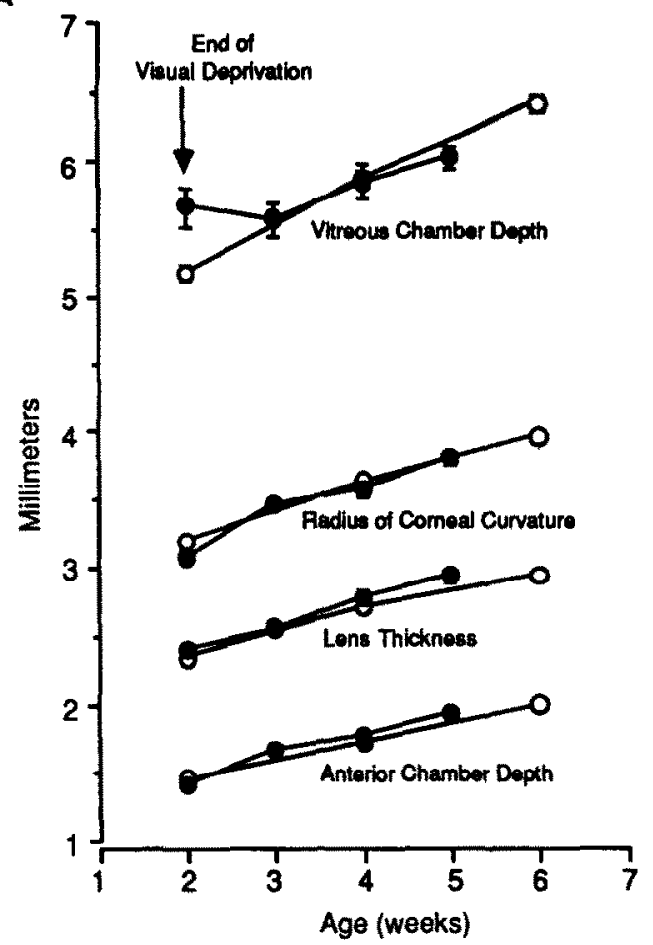

B

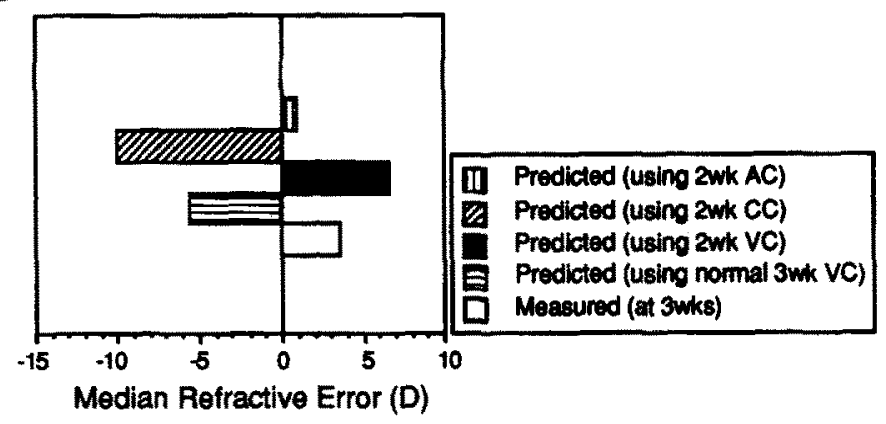

Fig. 2. (A) Mean ( \pm I SE) changes of ocular components in eyes recovering from visual deprivation myopia shown in Fig. 1. Standard errors are smaller than data points unless indicated. Solid circles represent eyes initially made myopic by visual form deprivation, open circles represent measurements from normal controls. For both groups, paired $t$-tests were used to assess age-related morphological changes. The anterior chamber depth includes the thickness of the cornea, estimated to be about $0.24 \mathrm{~mm}$. Corneal curvature is expressed as the radius of curvature; larger values indicate less curved corneas. (B) An estimation of the relative effects of changes in the different ocular components on the observed recovery from visual deprivation myopia between 2 and 3 weeks of age $(\mathrm{AC}=$ anterior chamber depth; $\mathrm{CC}=$ radius of corneal curvature; $\mathrm{VC}=$ vitreous chamber depth). The cessation of vitreous chamber growth, together with the normal growth of the anterior segment, is responsible for emmetropization from myopia. Predicted refractions were obtained from ray-tracing calculations using the measurements from individual 3-week-old eyes except for the parameter in parentheses. The discrepancy between the predicted and measured refractions gives an indication of the optical effect of the change in that parameter (see text for details). If, from 2 to 3 weeks of age, the vitreous chambers grew normally for their age, or if the corneal curvatures had not changed normally, the eyes would have remained myopic. The anterior chamber depth has a relatively smaller role in the determination of refractive state but also contributes to the recovery from visual deprivation myopia.

observed refractive state. Despite their role in recovery from myopia, anterior chamber depth and corneal curvature did not differ from normal controls so we are not inclined to consider them to be actively involved in the observed emmetropization.

Emmetropization from visual deprivation myopia is the combined result of the cessation 


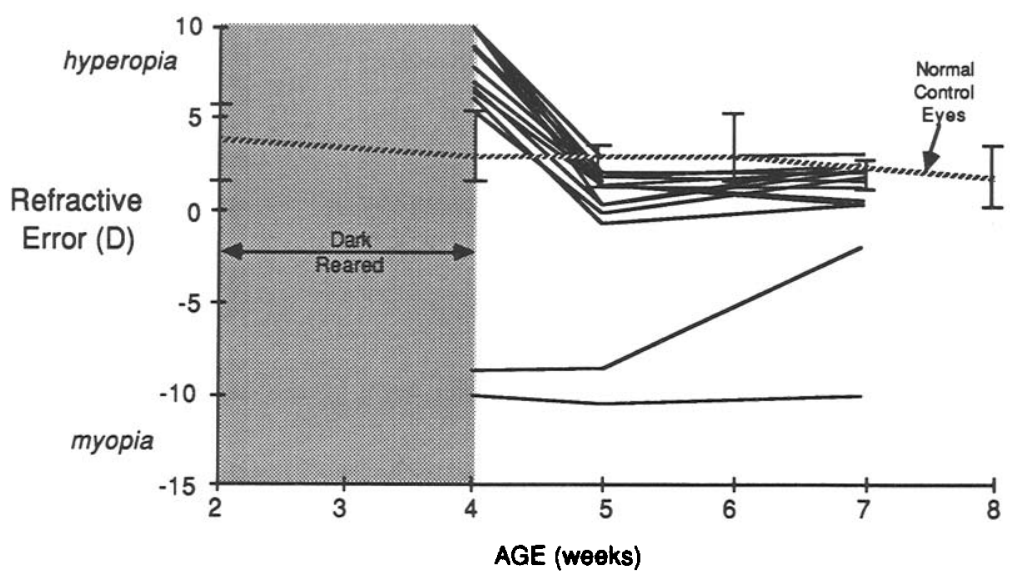

Fig. 3. Recovery from dark-induced hyperopia takes place after chicks are returned to normal conditions. The individual refractive errors of chicks reared in the dark until 4 weeks of age are shown as solid lines. Refractive error mesurements were made at 4 weeks when the chicks first came out of the dark, then at 5 and 7 weeks of age ( 1 and 3 weeks of normal rearing respectively). Because the question of interest here is emmetropization from hyperopia, the one dark-reared bird that was myopic in both eyes was omitted from the statistical analysis. The broken line shows the median refractive errors of normally-reared control chicks (same as in Fig. 1).

of vitreous chamber growth while the anterior segment continues to grow normally. Figure 2B shows that had the vitreous chambers in the emmetropizing myopic eyes continued to grow from 2 to 3 weeks at a rate equal to that of normal birds (thereby remaining longer than normal), the eyes would have been approx. $10 \mathrm{D}$ more myopic than observed (4th bar down). Had the vitreous chamber not grown at all while the rest of the eye continued to grow (3rd bar down), the refractive error would have been only slightly more hyperopic than that actually measured (bottom bar).

Recovery from hyperopia. Raising chicks in the dark for four weeks produced significant hyperopia* relative to normal 4-week-old controls $(+8.24$ vs $+2.60 \mathrm{D}$ respectively; $U$-test, $P<0.01$ ). Figure 3 shows that this hyperopia was abolished within 1 week after the chicks were returned to normal lighting conditions (4-5 weeks: +8.24 to $+1.24 \mathrm{D}$; Wilcoxon, $P<0.01)$. The refraction of recovering eyes overshot slightly the refractions of control eyes $(+1.24$ vs $+2.37 \mathrm{D}$ respectively; $U$-test, $P<0.01$ ), but 2 weeks later ( 7 weeks of age) the difference between experimental and control eyes was not significant $(+1.69$ vs $+2.09 \mathrm{D}$ respectively; $U$-test, $P=0.07$ ), nor was the

*For reasons that are unclear we find that among darkreared chicks about 1 in 10 become myopic rather than hyperopic. For completeness Figures 4 and 9 show 2 such outliers (4 eyes). Because we were interested in the ability of eyes to recover from hyperopia we omitted these outliers from the statistical analyses. difference in refractive error measured in the experimental eyes at 5 and 7 weeks $(+1.24$ vs $+1.69 \mathrm{D}$ respectively; Wilcoxon, $P=0.126$ ).

As in the recovery from visual deprivation myopia, recovery from dark-induced hyperopia is apparently the result of an active adjustment in vitreous chamber growth. In terms of the anatomical measurements made (Fig. 4), the growth toward emmetropia from dark-induced hyperopia can only be explained by the increase in vitreous chamber depth between 4 and 5 weeks $(6.69-6.98 \mathrm{~mm}$; paired $t$-test, $P<0.01)$. Although, the rate of vitreous chamber growth was not significantly greater than controls (Fig. 4B top; one-tailed unpaired $t$-test, $P=0.135$ ), the increase in vitreous chamber depth occurred despite the fact that at 4 weeks it was already significantly greater than normal (6.69 vs $5.86 \mathrm{~mm}$; $t$-test, $P<0.01$ ).

Unlike emmetropization from visual deprivation myopia, in which changes in corneal curvature, anterior chamber, and vitreous chamber all play a part, emmetropization from dark-induced hyperopia can only be explained by the observed increase in vitreous chamber growth. The increase in anterior segment depth and radius of corneal curvature during the first week after dark-rearing was discontinued (Fig. 4A) effectively reduce the total optical power of the eye. Specifically, during this period there were significant increases in the radius of corneal curvature $(3.98-4.09 \mathrm{~mm}$; paired $t$-test, $P<0.01)$, anterior chamber depth $(1.27-1.55 \mathrm{~mm}$; paired $t$-test, $P<0.01)$, and 


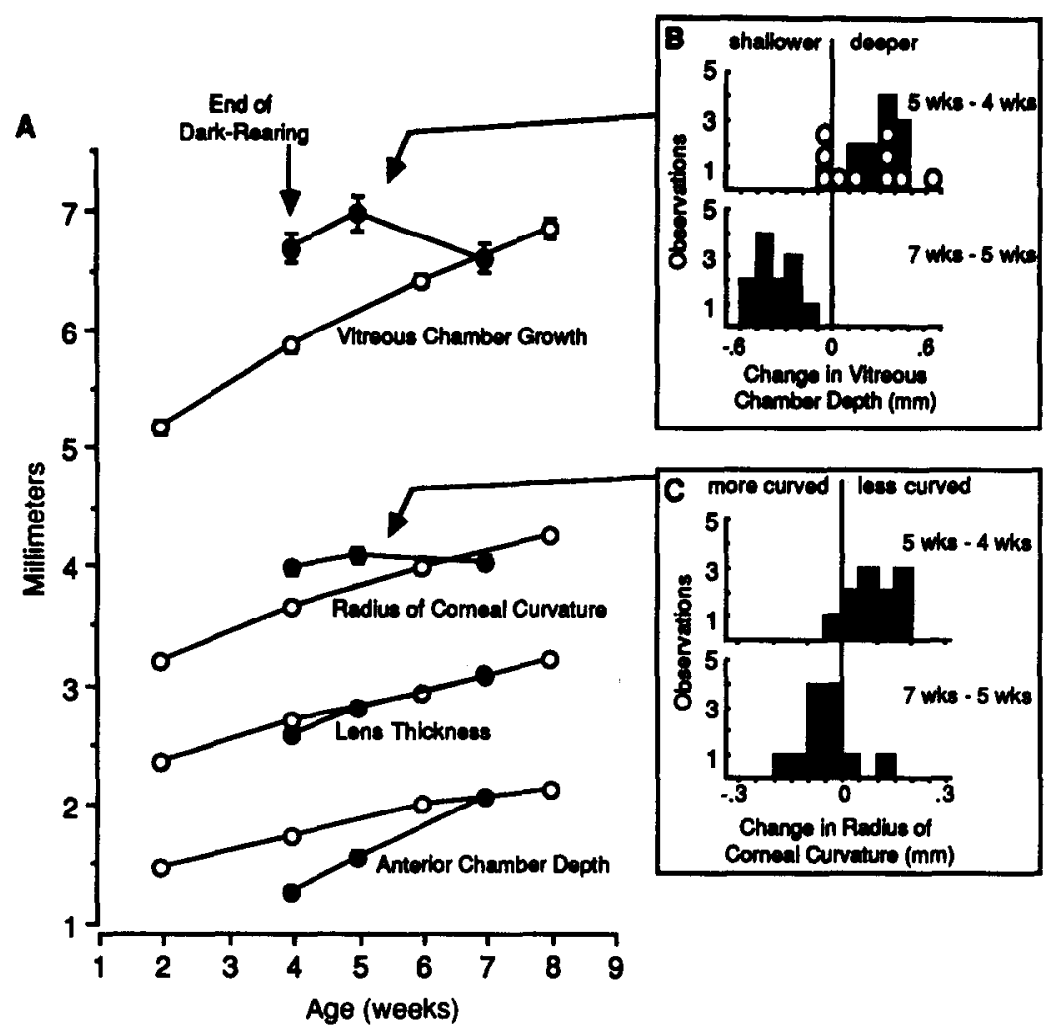

D

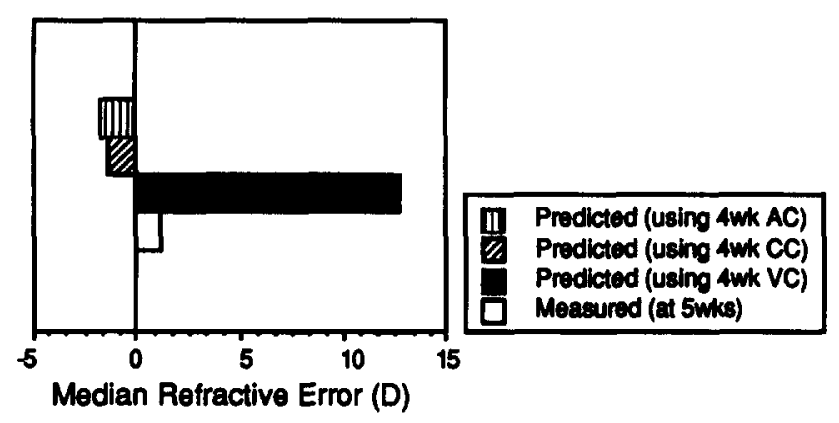

Fig. 4. (A) Mean ( $\pm 1 \mathrm{SE}$ ) changes of ocular components in eyes recovering from hyperopia shown in Fig. 3. Standard errors are smaller than data points unless indicated. Solid circles represent eyes initially made hyperopic by dark-rearing, open circles represent measurements from normal controls. (B) Histograms showing the increase in vitreous chamber depth of the experimental eyes between 4 and 5 weeks, and the decrease between 5 and 7 weeks (positive values indicate deepening vitreous chambers). Open circles in the top histogram show the normal growth of the vitreous chamber in 10 control eyes. The decrease in vitreous chamber depth between 5 and 7 weeks probably reflects a decreased rate of growth together with a concomitant increase in lens thickness. (C) Histograms showing the changes in the radius of corneal curvature of eyes recovering from dark-induced hyperopia (positive values indicate corneas becoming flatter). During the first week of recovery (4-5 weeks of age) the corneas become significantly less curved, which by itself would increased the hyperopia. Later (5.7 weeks of age) the corneas become significantly more curved. (D) An estimate of the relative effects of changes in the different ocular components on the observed recovery from dark-induced hyperopia between 4 and 5 weeks of age ( $A C=$ anterior chamber depth; $C C=$ radius of corneal curvature; $V C=$ vitreous chamber depth). Only the continued increased in vitreous chamber depth can explain the decrease in hyperopia observed; the other changes all reduce optical power and work against the recovery from hyperopia. Predicted refractions were obtained from ray-tracing calculations for the individual eyes using the measurements from individual 5-week-old eyes except for the parameter in parentheses which was held at its 4-week value. The discrepancy between the predicted and measured refractions indicates the optical effect of the change in that parameter (see text for details). Note that if the vitreous chamber depth did not increase but remained at the 4-week depth, the refraction would have remained severely hyperopic. The observed growth of the anterior segment does not contribute to the emmetropization from dark-induced hyperopia because the increases in radius of corneal curvature, anterior chamber depth, and lens thickness all reduce the optical power of the eye. This is shown by the fact that the predicted refractions based on the 4-week measurements of corneal curvature and anterior chamber depth are myopic. 
lens thickness $(2.59-3.08 \mathrm{~mm}$; paired $t$-test, $P<0.01$ ). Because these changes each reduce optical power, thereby increasing hyperopia, they cannot play a role in the recovery (Fig. 4D).

As described earlier ray-tracing was used on individual eyes to determine the role of the changes of each of the ocular components in the recovery from hyperopia. Each component, in turn, was held at its 4 week value (when dark-rearing ended and the eyes were still hyperopic). These calculations show clearly that if the increase in depth of the vitreous chamber between 4 and 5 weeks did not occur the eyes would have remained severely hyperopic (Fig. 4D). Further, if the corneal curvatures or anterior chamber depths remained at their 4 week values, the optical power of the eye would have been too great for its length and myopia would result. In other words, between 4 and 5 weeks the observed change in the growth of the anterior segment actually reduces optical power and works against the return to emmetropia from hyperopia, thereby necessitating even greater increases in vitreous chamber depth than if the anterior segment did not change.

During the second and third weeks following the return to normal rearing conditions (5-7 weeks of age), even though the eyes had achieved relatively normal refractions, the ocular components were still undergoing readjustment. Figure $4 \mathrm{C}$ shows that corneal curvature (which had continued to decrease during the first week of emmetropization) reverses its direction of change during the second and third weeks showing a small, but significant, decrease in radius of curvature ( 4.09 vs $4.03 \mathrm{~mm}$; paired $t$-test, $P<0.05$ ). This late-onset readjustment in the shape of the anterior segment is also seen in the anterior chamber depth which does not completely return to normal levels until after three weeks of normal rearing conditions.

The significant decrease in the vitreous chamber depth between 5 and 7 weeks $(6.98-6.61 \mathrm{~mm}$; paired $t$-test, $P<0.01$; see Fig. 4B) is most likely the combined result of a reduction in vitreous chamber growth and a simultaneous increase in lens thickness. There is, in fact, a significant increase in the thickness of the lens $(2.81-3.08 \mathrm{~mm}$; paired $t$-test, $P<0.01)$ during the 5-7 week period when the vitreous chamber depth is significantly reduced. The increased lens thickness $(0.27 \mathrm{~mm})$ does not completely account for the decreased vitreous chamber depth $(0.37 \mathrm{~mm})$ suggesting that a shift in the position of the lens may also occur.

\section{Optic nerve section and emmetropization}

The recovery paradigm was used on 15 opticnerve-sectioned chicks ( 7 myopic, 8 hyperopic) to determine whether the brain is involved in experimental emmetropization. After the visual manipulations were discontinued growth in the direction of emmetropia was observed despite the optic nerve section, although this growth was not properly controlled and emmetropia was not maintained (Fig. 5). One week after the end of the visual deprivation the refractive errors of the optic-nerve-sectioned eyes had changed significantly $(-14.95$ to $+4.0 \mathrm{D}$; Wilcoxon, $P<0.05$ ). Similarly, eyes made hyperopic by 4 weeks of dark-rearing, then allowed normal visual conditions, also changed significantly toward emmetropia despite the optic nerve section (Fig. 5, right; +6.1 to $+2.3 \mathrm{D}$; Wilcoxon, $P<0.05$ ). A difficulty in this experiment is the fact that after 1 week of partial visual form deprivation, the myopia in eyes with optic nerve section (Fig. 5, top left) is significantly greater than the $-4.41 \mathrm{D}$ produced in the visually form deprived contralateral eyes without optic nerve section (Fig. 5, bottom left; Wilcoxon, $P<0.05$ ). This difference may be because the optic-nerve-sectioned eye was not adducted to look through the occluder's opening and thus received a relatively greater visual deprivation (see Troilo et al., 1987).

Because the unoperated eyes of these birds had the same sign of refractive error as the optic-nerve-sectioned eyes, the possibility exists that the unoperated eye guides emmetropization in both eyes. To test this 4 chicks were raised with visual deprivation of the optic nervesectioned eye while the unoperated eye remained unoccluded. Figure 6 shows that after 2 weeks of monocular visual deprivation, the optic-nerve-sectioned eyes were myopic while the contralateral eyes were approximately emmetropic (means: -6.8 vs $+1.0 \mathrm{D}$ respectively). After the visual deprivation was discontinued at 2 weeks of age the opticnerve-sectioned eyes were able to grow back toward emmetropia $(2$ weeks $=-6.8 \mathrm{D} ; 3$ weeks $=-0.71 \mathrm{D}$; and 4 weeks $=+5.39 \mathrm{D})$ despite the obvious difference in the refractions between the unoperated and optic-nervesectioned eye.

Although initially the optic-nerve-sectioned eyes respond appropriately to refractive errors, they eventually overshoot emmetropia resulting 


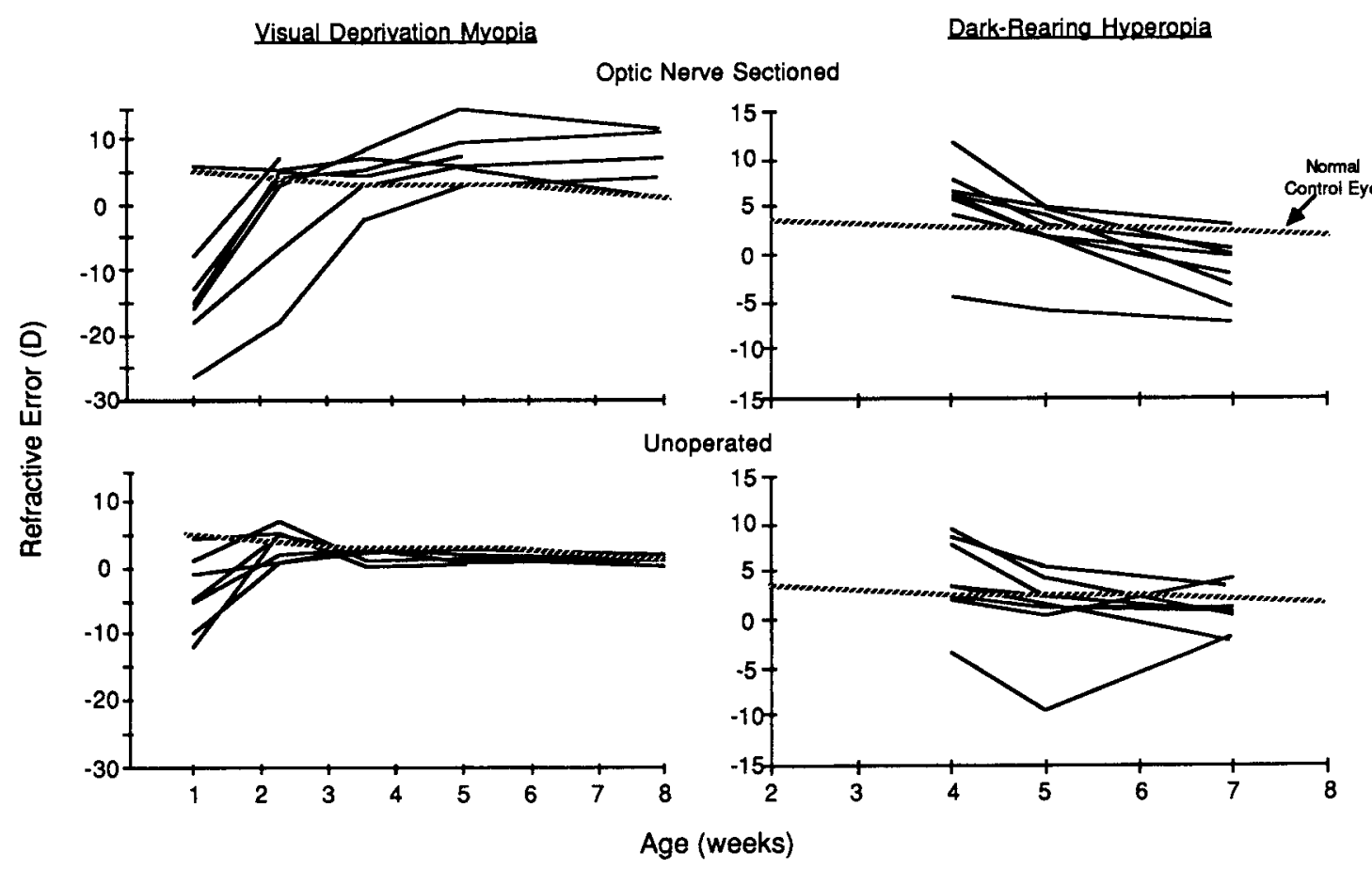

Fig. 5. Eyes with optic nerve section (top), and their contralateral unoperated control eyes (bottom), made either myopic by 1 week of partial visual form deprivation (left) or hyperopic by 4 weeks of dark-rearing (right), respond with the appropriate compensatory changes and bring about growth toward emmetropia. The optic nerves were cut at hatching for the visual form deprivation group, and at 4 weeks for the dark-rearing group. In both groups the nerve-sectioned eyes grow significantly past emmetropia resulting in a refractive error with a sign opposite to that initially induced. The dashed lines represent the median refractive errors of untreated control eyes measured at 1,2, 4,6 and 8 weeks of age (same as Figs 1 and 3). As in Fig. 3, the one dark-reared bird that was myopic in both eyes was omitted from the statistical analyses.

in a reversal of the original refractive error (Figs 5 and 6). Four weeks after the visual form deprivation was discontinued, the initially

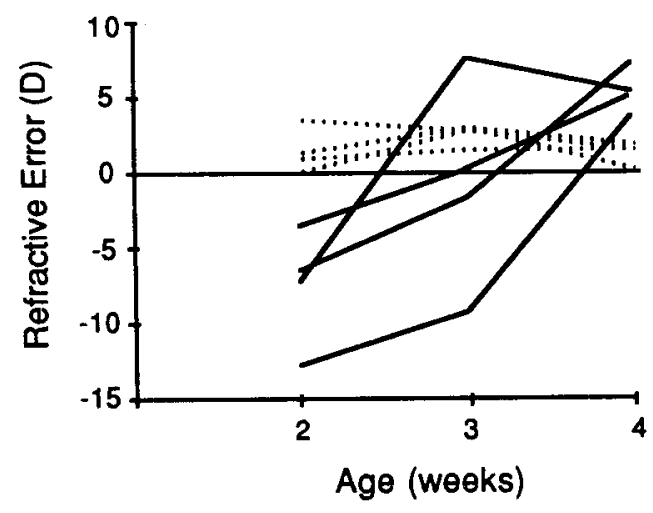

Fig. 6. Recovery from monocularly induced visual deprivation myopia in optic-nerve-sectioned eyes shows that the refractive state of the unoperated eye does not guide the growth of the optic-nerve-sectioned eye. Eyes with optic nerve section (solid lines) grow toward, and eventually overshoot, emmetropia even when the contralateral unoperated eyes (dashed lines) possess normal refractions. The optic-nerve-sectioned eyes were deprived of form vision in the lateral visual field from hatching until 2 weeks of age. Refractometry was performed at 2, 3 and 4 weeks of age. myopic eyes with optic nerve section became significantly hyperopic relative to the contralateral control eyes $(+6.93$ vs $+1.69 \mathrm{D}$, Wilcoxon, $P<0.02$ ). Conversely, three weeks after the dark-reared hyperopic chicks were returned to normal lighting conditions, the optic-nerve-sectioned eyes became significantly myopic relative to the contralateral control eyes $(-1.5$ vs $+0.5 \mathrm{D}$, Wilcoxon, $P<0.05)$.

The reversal of refractive error in optic-nervesectioned eyes recovering from induced refractive error corresponds with small errors in the regulation of vitreous chamber growth (Fig. 7). On average, the initial change in vitreous chamber growth of optic-nerve-sectioned eyes (which correct the induced refractive error) continue and contribute to the development of the refractive error opposite in sign. Figure 7 shows that the visually form-deprived optic-nervesectioned eyes have longer vitreous chambers than their fellow control eyes at 1 week, reflecting the significantly greater myopia in these eyes at that age (see Fig. 5, left). After removal of the occluders, when the refractions of the opticnerve-sectioned eyes moved toward emmetropia 


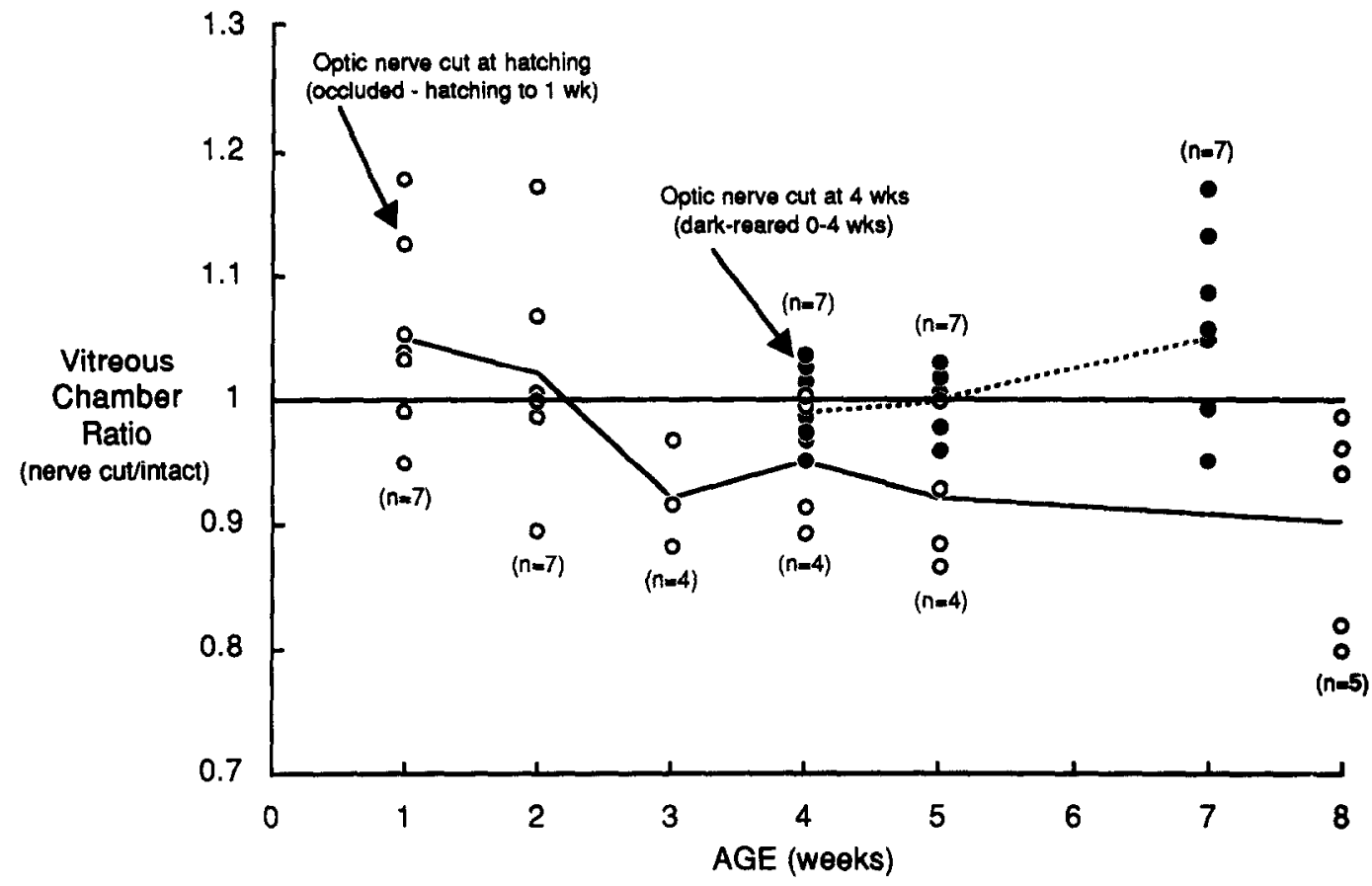

Fig. 7. The change in vitreous chamber depth of optic-nerve-sectioned myopic and hyperopic eyes relative to their contralateral control eyes suggests that inappropriately regulated vitreous chamber growth may be responsible for the development of refractive errors opposite to those initially induced. This figure shows the change in the ratio of the vitreous chamber depth of optic-nerve-sectioned eyes to the vitreous chamber depth of the contralateral unoperated eyes. Open circles represent individual chicks undergoing visual form deprivation for 1 week. Solid circles show the chicks that were reared in complete darkness until 4 weeks of age. Lines show the means of the ratios at different ages (solid line for initially myopic eyes; dashed line for initially hyperopic eyes). Beginning 1 week after optic nerve section, the change in vitreous chamber growth in response to the ametropia is exaggerated in the optic-nerve-sectioned eye relative to the unoperated fellow eye. Compared to their respective unoperated fellow eyes the growth rate is, on average, greater in the operated hyperopic eyes and less in the operated myopic ones. The ratios of vitreous chamber depth of the dark-reared eyes begin at 1 because both eyes had identical treatments to that point; in addition, at 5 weeks both the unoperated and optic-nerve-sectioned eyes had grown to emmetropia from hyperopia and the refractions were not significantly different. The ratios of vitreous chamber depth of the visually form deprived eyes began greater than 1 because the optic-nerve-sectioned eyes had significantly longer vitreous chambers and more myopia than the unoperated eyes.

and then into hyperopia, the vitreous chambers of the optic-nerve-sectioned eyes grew less than the control eyes as seen by the change in the ratios of experimental over control vitreous chamber depths from 2 to 8 weeks (means: $1.02-0.90$, respectively; one-tailed paired $t$-test, $P<0.025$ ). Both the refractions and the vitreous chamber depths of the dark-reared chicks were the same in both eyes after the chicks were removed from the dark at 4 weeks of age when the chicks received unilateral optic nerve section. After 1 week of normal visual experience both the refractions and vitreous chamber depths of both the optic-nerve-sectioned and the unoperated fellow eyes had returned to control levels. However, by the end of the third week in the normal visual environment, a slight excess of vitreous chamber growth in the optic-nerve- sectioned eyes can be seen in the increase of the vitreous chamber ratios from 5 to 7 weeks (means: $1.0-1.05$, one-tailed paired $t$-test, $P<0.03)$.

As in unoperated eyes recovering from darkinduced hyperopia, the mean corneal curvature of optic-nerve-sectioned eyes was initially flatter than normal (radius of curvature: $4.27-4.09 \mathrm{~mm}$ respectively; paired $t$-test, $P<0.01$ ), but eventually became more curved and approached normal values $(4.27-4.18 \mathrm{~mm}$; paired $t$-test, $P<0.05$ ). Such an increase in corneal curvature increases the total optical power and, together with the concomitant vitreous chamber elongation, contributes to the overshoot into myopia measured in optic-nerve-sectioned eyes at 7 weeks of age. Because this adjustment in corneal shape occurs in optic-nerve-sectioned 
eyes the mechanism must be located within the eye. Also as in the unoperated eyes this adjustment in corneal shape lags slightly behind the refractive change, and is in the wrong direction to account for the recovery from hyperopia.

\section{DISCUSSION}

In this paper we present an experimental study of emmetropization in chicks. After refractive errors were induced (myopia by deprivation of form vision, hyperopia by dark-rearing) the normal visual environment was restored and the changes in the eye were monitored. Eyes returned quickly to emmetropia mainly by modulating the growth rate of the vitreous chamber. The anterior segment also changed in the direction of normal values, but these changes began after the vitreous chamber changes and continued after emmetropia had been attained.

We suggest that there are two mechanisms controlling eye growth in chicks; one uses a visual signal related to refractive error and guides the growth of the vitreous chamber toward achieving emmetropia, the other is a nonvisual mechanism related to the shape of the eye and results in changes toward normal size and shape. Both of these mechanisms appear to operate to some degree in optic-nerve-sectioned eyes implying that both are located within the eye itself. Emmetropia is not attained in opticnerve-sectioned eyes however; these eyes overshot emmetropia and reversed the sign of the initial refraction error.

\section{Visual factors in experimental emmetropization}

Visual control of eye growth in the chick is shown by the appropriate modulation of vitreous chamber growth in response to myopic or hyperopic refractive error. In recovery from hyperopia the growth of the vitreous chamber continues, while in eyes recovering from myopia the vitreous chambers stop growing. Because this compensation occurs despite the fact that the vitreous chambers are abnormally enlarged in both cases, eye size cannot be the error signal controlling the compensatory change.

We view the experiments presented here and the lens-rearing experiments of Schaeffel et al. (1988) as being complementary. In our experiments, eyes with refractive errors and abnormal shapes grow back toward emmetropia and normal shape; in the lens-rearing experiments the eyes grow away from normal shape to compensate for an artificially imposed refractive error. In both experiments the eye responds to an imposed refractive error by appropriately adjusting the growth of the vitreous chamber to reduce the refractive error.

The mechanism by which visual regulation takes place appears to be largely located within the eye itself. Recent studies indicate that even local regions of the retina must be able to modulate the growth of the vitreous chamber in response to visual experience in that region. Chicks deprived of form vision in part of the visual field develop enlarged vitreous chambers and myopia restricted to the deprived region of the retina (Wallman et al., 1987). Furthermore, the localized effects of partial visual form deprivation are still manifest in eyes with optic nerve section (Troilo et al., 1987). In normal eyes emmetropization may also be locally controlled. Miles and Wallman (1990) report that when only the ventral retina is made functionally hyperopic by rearing chicks in an environment with a patterned ceiling kept just above the head, the ventral part of the eye enlarges and becomes myopic relative to controls, thereby partially compensating for the imposed hyperopia.

The possibility that the brain is involved in the regulation of eye growth cannot be ruled out. Although eyes with optic nerve section are also able to sense the sign of an induced refractive error and initiate the proper compensatory changes, these responses are not properly controlled; the eye grows past emmetropia to the opposite refractive error. Furthermore, an intact optic nerve is required for normal eye growth; the eyes in which the optic nerve is cut are smaller and more hyperopic than normal eyes (Troilo et al., 1987). These results suggests that either the loss of brain-mediated visual processes or the loss of retinal ganglion cells affects the fine control of vitreous chamber growth and refractive state. In recovery from both myopia and hyperopia, the overshoot in emmetropization appears to be caused by too strong a response in vitreous chamber growth to the initial refractive error. If the vitreous chamber growth response is proportional to the magnitude of the refractive error, this may be because the refractive error detecting circuitry is sluggish; if the growth response depends only on the sign of the refractive error, it may be that the refractive error detecting circuitry is less sensitive.

Accommodation is a plausible brain-mediated visual function for the control of eye growth 
because it can sense refractive state and respond with direct mechanical influences on the eye. In order to examine the role of accommodation in the regulation of eye growth, in recent studies we have used lesions of the Edinger-Westphal nucleus to block the motor output of the accommodation system. Apparently the EdingerWestphal nucleus is not necessary for either the induction of visual deprivation myopia or for the recovery from induced refractive errors (Troilo \& Wallman, 1988; Troilo, 1989). Furthermore, lesions of the Edinger-Westphal nuclei do not block the compensation for lens-imposed refractive errors (Schaeffel, Troilo, Wallman \& Howland, 1990). These results suggest that accommodation is not necessary for the regulation of eye growth, and do not support the commonly held assumption that refractive changes associated with near vision are due to accommodation. There is, however, evidence that bilateral lesions of Edinger-Westphal have a transient effect on refractive state and increase the inter-individual variability of all refractions (Schaeffel et al., 1990; Troilo, 1989).

Even if the action of accommodation is not involved in the control of eye growth the stimuli that drive accommodation may also drive the visually guided emmetropization mechanism, although the action of accommodation would reduce the error signals used for emmetropization. At present we can only speculate on the possible neural processes used to avoid this problem.

In an earlier study (Wallman, Rosenthal, Adams \& Romagnano, 1981b), the amount of myopia induced by visual form deprivation was reduced by cutting the short ciliary nerves, and thereby blocking the final motor pathway of accommodation. Recently, we observed that removal of the ciliary ganglion did not always reduce the development of induced myopia, nor did it block the recovery from it (unpublished results). The reasons for this difference are unclear and require further investigation.

\section{Evidence for nonvisual regulation}

Three lines of evidence argue that, in addition to the visually guided control of eye growth just

*We refer to the shape-related mechanism as any morphological change other than the visually guided change in vitreous chamber depth. The putative mechanism may involve a complicated interaction of components such as the relationship of corneal curvature to axial length, and may be due to mechanical effects and not necessarily to feedback regulation in the formal sense. discussed, there exists a mechanism of eye growth that is related to the shape of the eye.* First, in the experiments on recovery from dark-induced hyperopia, after normal refractions are attained the anterior segment continues to change until normal age-related dimensions are met. The cornea becomes more curved and the anterior chamber deepens, both changes returning the eye to normal shape. Furthermore, the corneal change is in the direction opposite to both that seen in normal growth and in eyes recovering from myopia. Second, two distinct hyperopic conditions can exist in birds with severed optic nerves. In birds without visual deprivation the eyes are slightly shorter than normal but otherwise normal in shape (similar to neonates), and they remain stably hyperopic (Troilo et al., 1987). In contrast, the eyes of dark-reared optic-nervesectioned birds described in this paper are enlarged, but have flat corneas and shallow anterior chambers. When returned to normal conditions these eyes grow in length, quickly losing their hyperopia, and subsequently show increases in corneal curvature and anterior chamber depth. It would seem that the only difference between these two types of hyperopia is that in the dark-reared eyes there is an abnormal relation of eye size to anterior chamber shape, which is eventually corrected. Third, in situations in which eye shape and refraction are both abnormal, complete compensatory changes occur. Specifically, complete recovery from induced refractive error in chicks occurs extremely rapidly; tens of diopters of change in refractive error occur within the first week of normal visual conditions. Here both the refractions and the eye shape are at first abnormal, and so both the visually guided and shape-related mechanisms are presumably making corrections. In contrast, in spectacle lens experiments the compensatory responses are not complete (Schaeffel et al., 1988). Perhaps this is because in this situation the visually guided mechanism promotes growth toward an abnormal shape to compensate for the imposed refractive error while the shape-related mechanism acts to maintain the normal shape (i.e. the visually guided and shape-related mechanisms work in opposing directions). Because this condition effectively results in growth toward emmetropia but away from normal shape, we suggest that the visually guided mechanism is more potent than the shape-related one. However, a recent report by Schaeffel and Howland 
(1991) suggests that when shape, but not refraction, is abnormal the shape-related mechanism may override visual regulation. Schaeffel and Howland corrected induced refractive errors in chicks with the appropriate spectacle lenses. Despite the apparent elimination of the visual error signal, in some of the chicks the refractions continued to change toward normal (uncorrected) levels.

We are not inclined to view the shape-related mechanism as a special part of emmetropization. Rather, it may be part of the morphogenic regulatory mechanisms of the body that, together with mechanical constraints, make eyes eye-shaped, kidneys kidney-shaped, etc. Although little is known about these regulatory processes, the shape-related control of eye growth is probably related to organogenesis because the tissues of the growing eye must continually be remodeled for normal growth to occur. The mechanism resulting in the shape of the eye may only approximately maintain the proper optical relationships between the refracting surfaces; it would then be the function of the visually guided growth-control mechanism to achieve the refractive state adaptive to the eye's particular visual experience.

\section{CONCLUSION}

Experimental emmetropization-the ocular growth adjustments leading toward emmetropia from induced refractive errors - can be described as occurring by two mechanisms. The faster modulates vitreous chamber growth and is guided by refractive state, and the slower produces anterior segment changes and is related to the shape of the eye. These two mechanisms of eye growth control normally work together to achieve and maintain emmetropia as the eye grows during its normal development. The non-visual shape-related mechanism may keep the ocular components in approximately the proper proportions while the visual refraction-sensitive mechanism adjusts the vitreous chamber depth, thereby positioning the retina at the distance from the optics best suited for the eye's visual experience. We suggest that the visual mechanism is responsible for the emmetropization observed in the axial refractions of normal eyes typically viewing objects at a range of distances, and is also responsible for the control of local refractive differences within the eye (e.g. Fitzke, Hayes, Hodos, Holden \& Low, 1985; Miles \& Wallman, 1990). Finally, to some degree both mechanisms of eye growth occur within the eye itself, but the optic-nervesection experiments presented in this paper suggest that brain-mediated visual processes may also be involved in emmetropization. The experimental separation of the various eye growth mechanisms identified here presents a challenge for future work in this area.

Acknowledgements - The authors would like to thank Drs W. Hodos, H. C. Howland, M. D. Gottlieb and F. A. Miles for their comments and suggestions on the dissertation upon which this paper is based. Thanks also to D. Nickla, and Dr S. J. Judge for their comments on an earlier version of the paper. Some of the data shown in Fig. 5 were collected with the assistance of M. D. Gottlieb. The normal data in Fig. 4B were provided by A. Xu and L. Marran. This research was supported by the National Institutes of Health research grant EY-02727.

\section{REFERENCES}

van Alpen, G. W. H. M. (1961). On emmetropia and ametropia. Ophthalmologica (Suppl.), 142, 1-92.

Banks, M. S. (1980). Infant refraction and accommodation. In Soko, S. (Ed.), Electrophysiology and psychophysics. Their use in opthalmic diagnosis (pp. 205-232). Boston: Little, Brown \& Co.

Borish. I. M. (1970). Clinical refraction. Chicago: Professional Press.

Donders, F. C. (1952). Accommodation and refraction of the eye. London: New Syndenham Society.

Duke-Elder, W. S. (1930). An investigation of the effect on the eyes of occupations involving close work. British Journal of Ophthalmology, 14, 609-620.

Dunphy, E. B., Stoll, M. R. \& King, S. H. (1968). Myopia among male graduate students. American Journal of Ophthalmology, 65, 518-521.

Fitzke, F. W., Hayes, B. P. Hodos, W., Holden, A. L. \& Low J. C. (1985). Refractive sectors in the visual field of the pigeon eye. Journal of Physiology, London 369, 33-44.

Glickstein M. \& Millodot, M. (1970). Retinoscopy and eye size. Science, 168, 605-606.

Goldschmidt, E. (1968). On the etiology of myopia: An epidemiological study. Copenhagen: Munksgaard.

Gottlieb, M. D., Fugate-Wentzek, L. A. \& Wallman, J. (1987). Different visual deprivations produce different ametropias and different eye shapes. Investigative Ophthalmology and Visual Science, 28, 1225-1235.

Hoyt, C. S., Stone, R. D., Fromer, C. \& Billdon, F. A. (1981). Monocular axial myopia associated with neonatal eyelid closure in human infants. American Journal of Ophthalmology, 91, 197-200.

Mark, H. H. (1972). Emmetropization: Physical aspects of a statistical phenomenon. Annals of Ophthalmology, 4, 393-401.

Marsh-Tootle, W. L. \& Norton, T. T. (1989). Refractive and structural measures of lid-suture myopia in tree shrew. Investigative Ophthalmology and Visual Science, 30 2245-2257.

McBrien, N. A. \& Barnes, D. A. (1984). A review and evaluation of theories of refractive error development. Ophthalmic and Physiological Optics, 4, 201-213. 
McBrien, N. A. \& Norton, T. T. (1987). The development of ocular growth and refractive state in normal and monocularly deprived tree shrews (Tupaia belangeri). Society for Neurosciences Abstracts, 13, 1535.

Medina, A. (1987a). A model for emmetropization: Predicting the progression of ametropia. Ophthalmologica, 194, 133-139.

Medina, A. (1987b). A model for emmetropization: The effect of corrective lenses. Acta Ophthalmologica, 65 , 565-571.

Miles, F. A. \& Wallman, J. (1990). Local ocular compensation for imposed local refractive error. Vision Research, 30, 339-349.

Mohindra, I. \& Held, R. (1981). Refraction in humans from birth to five years. In Fledelius, H. C., Alsbrik, P. H. \& Goldschmidt, E. (Eds.), Proceedings of the third international myopia conference (pp. 19-27). Documenta Ophthalmologica Proceedings Series, 28. The Hague, Netherlands: Junk.

Morgan, M. W. (1967). A review of the major theories for the genesis of refractive state. In Hirsch, M. J. (Ed.), Synopsis of the refractive state of the eye: $A$ symposium (pp. 8-12). American Academy of Optometry Series, 5 .

Nathan, J., Kiely, P. M., Crewther, S. G. \& Crewther, D. P. (1985). Disease-associated visual degradation and spherical refractive errors in children. American Journal of Optometry and Physiological Optics, 62, 680-688.

O'Leary, D. J. \& Millodot, M. (1979). Eyelid closure causes myopia in humans. Experientia, 35, 1478-1479.

Raviola, E. \& Wiesel, T. N. (1985). An animal model of myopia. New England Journal of Medicine, 312, 1609-1615.

Richler, A. \& Bear, J. C. (1980). Refraction, nearwork and education. A population study in Newfoundland. Acta Ophthalmologica, 58, 468-478.

Robb, R. M. (1977). Refractive errors associated with hemangiomas of the eyelids and orbit in infancy. American Journal of Ophthalmology, 83, 52-58.

Rose, L., Yinon, U. \& Belkin, M. (1974). Myopia induced in cats deprived of distance vision during development. Vision Research, 14, 1029-1032.

Schaeffel, F. \& Howland, H. C. (1991). Properties of the feedback loops controlling eye growth and refractive state in the chicken. Vision Research, 31, 717-734.

Schaeffel, F., Glasser, A. \& Howland, H. C. (1988). Accommodation, refractive error and eye growth in chickens. Vision Research, 28, 639-657.

Schaeffel, F., Troilo, D., Wallman, J. \& Howland, H. C. (1990) Developing eyes that lack accommodation grow to compensate for imposed defocus. Visual Neuroscience, 4, $177-183$.
Sorsby, A. (1979). Biology of the eye as an optical system. In Safir, A. (Ed.), Refraction and clinical optics. Philadelphia: Harper \& Row.

Troilo, D. (1989). The visual control of eye growth in chicks. Doctoral Dissertation, City University of New York.

Troilo, D. \& Wallman, J. (1988). Experimental emmetropization in chicks. Investigative Ophthalmology and Visual Science (ARVO Suppl.), 29, 76.

Troilo, D., Gottlieb, M. D. \& Wallman, J. (1987). Visual deprivation causes myopia in chicks with optic nerve section. Current Eye Research, 6, 993-999.

Troilo, D., Judge, S. I., Ridley, R. \& Baker, H. (1990). Myopia induced by brief visual deprivation in a new world primate-the common marmoset (Callithrix jacchus). Investigative Ophthalmology and Visual Science (ARVO Suppl.), 28, 1246.

Wallman, J. \& Adams, J. I. (1987). Developmental aspects of experimental myopia in chicks. Vision Research, 27, 1139-1163.

Wallman, J., LeDoux, C. \& Friedman, M. B. (1978a). Simple devices for restricting the visual fields of birds. Behavioural Research Methods and Instrumentation, 10, 401-403.

Wallman, J., Turkel, J. \& Trachtman, J. (1978b). Extreme myopia produced by modest change in early visual experience. Science, 201, 1249-1251.

Wallman, J., Adams, J. \& Trachtman, J. N. (1981a). The eyes of young chickens grow toward emmetropia. Investigative Ophthalmology and Visual Science, 20, 557-561.

Wallman, J., Rosenthal, D., Adams, J. I. \& Romagnano, L. (1981b). Role of accommodation and developmental aspects of experimental myopia in chicks. In Fledelius, H. C., Alsbirk, P. H. \& Goldschmidt, E. (Eds.), Proceedings of the third international myopia conference (pp. 197-206). Documenta Ophthalmologica Proceedings Series, 28. The Hauge, Netherlands: Junk.

Wallman, J., Gottlieb, M. D., Rajaram, V. \& FugateWentzek, L. (1987). Retinal control of myopia and eye growth. Science, 237, 73-77.

Wiesel. T. N. \& Raviola, E. (1977). Myopia and eye enlargement after neonatal lid fusion in monkeys. Nature, London, 266, 66-68.

Wildsoet, C. F. \& Pettigrew, J. D. (1988). Experimental myopia and anomalous eye growth patterns unaffected by optic nerve section in chickens: Evidence for local control of eye growth. Clinical Vision Sciences, 3, 99-107.

Young, F. A. (1961). The effect of restricted space on the primate eyc. American Journal of Ophthalmology, 52 , 799-806.

Young, F. A. (1977). The nature and control of myopia. Journal of the American Optometric Association, 48, $451-457$. 